\title{
Protection des Vals de Loire en aval de la confluence avec la Vienne
}

\author{
Flood protection in the Loire valley \\ downstream Vienne confluence
}

A. Guilbot J.-C. Hémain P. Raous

Laboratoire d'hydrologie mathématique, Montpellier

\author{
G. Tessier \\ Direction départementale \\ du Loiret
}

J.-P. Kryn, Z. Gazowski

Service hydrologique centralisateur,
Le Val d'Authion, avec ses 30000 hectares de cultures exceptionnellement riches (fleurs, pépinières en particulier) est une des pièces mâtresses de l'économie du département du Maine \& Loire.

Or, ce Val, situé en rive droite de la Loire, est en dépression par rapport au lit de celle-ci. Il en est séparé et protégé par une levée de terre, construite au xII' siècle, et depuis lors constamment confortée, plusieurs fois exhaussée, pour la rendre insubmersible (des travaux sont en cours pour mettre son sommet en tous points à $1,10 \mathrm{~m}$ au-dessus des plus hautes eaux connues).

Les dernières ruptures de cette levée par des crues remontent à février 1711 et juin 1856 .

\begin{abstract}
Néanmoins, les populations et les élus, sensibilisés par des crues récentes, et conscients du fait qu'en 1856 les maximums des crues de la Loire et de la Vienne n'étaient pas simultanés, sont apparus préoccupés par l'éventualité d'une concomitance los d'une prochaine crue de type 1856 , conduisant à des débits bien supérieurs au maximum connu, et mettant en danger, non seulement le Val d'Authion, mais également tout le lit jusqu'à l'estuaire.

Le rapport Chapon (décembre 1979) signalait à ce sujet que "bien que des craintes aient été exprimées par les responsables politiques et économiques de la Basse Loire, il n'existe pas de traces écrites permettant de penser que les crues de la Vienne, qui sont importantes (elles peuvent
\end{abstract}

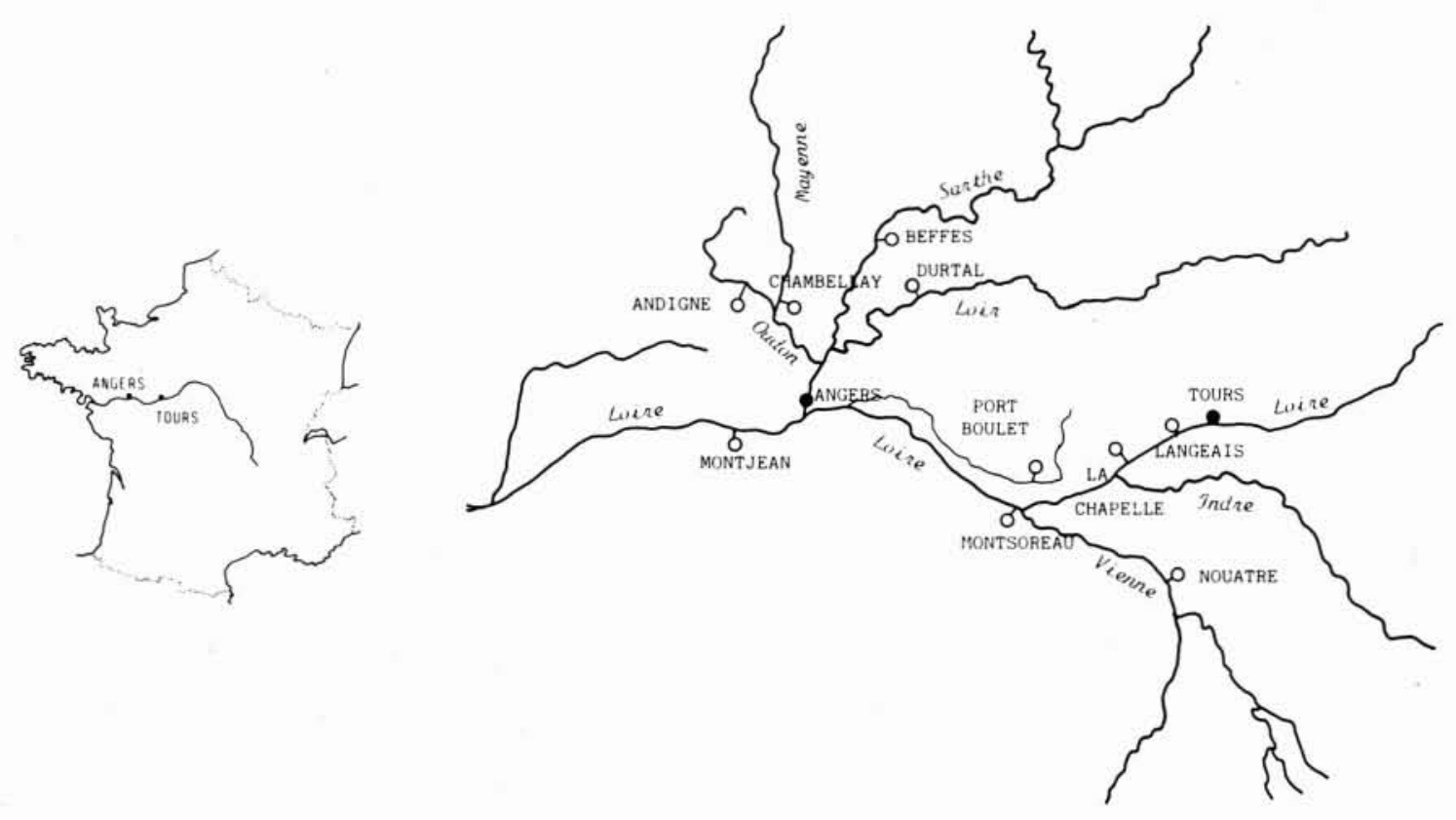

LA HOUILLE BLANCHE/ Nol/2-1984 
dépasser $3000 \mathrm{~m}^{3} / \mathrm{s}$ ), risquent de se conjuguer avec une forte crue de la Loire ", et de poursuivre : «... On ne saurait donc prévoir aujourd'hui au programme des travaux la réalisation d'ouvrages écrêteurs de crues sur la Vienne, alors qu'on ne sait même pas s'il en est besoin...».

Afin de répondre à ces interrogations, trois finalités avaient été fixées à l'étude confiée au Laboratoire d'hydrologie mathématique (LHM) :

- étude du risque de simultanéité des crues de la Loire et de la Vienne au niveau de leur confluence, lors des très fortes crues de l'une et de l'autre, et le risque de submersion de la levée d'Authion;

- étude du risque de submersion des vals situés en aval du confluent de la Maine, en simulant les débits du bassin intermédiaire;

- étude sommaire de l'efficacité sur la crue de la Loire d'un réservoir d'écrêtement simulé, situé sur la Vienne en aval du confluent de la Creuse.

Il s'agissait d'examiner l'existence de ces deux types de concomitance et les conséquences sur l'importance des crues de la Loire à Montsoreau.

Il y a crue de la Vienne sur 54 des 66 évènements dont nous disposions, c'est-à-dire dans $82 \%$ des cas. Sur ces 54 évènements, on peut retenir 72 pointes de crue distinctes sur la Vienne et faire correspondre à une pointe de crue de la Loire amont à une pointe de crue de la Vienne dans 60 cas. Pour certains d'entre eux, la notion de correspondance est délicate et l'on peut observer, pour un même événement présentant une succession de crues des décalages différents selon la pointe considérée.

La variable étudiée est le décalage en jours entre les débits de pointe aux stations de la Loire amont, et de la Vienne à Nouatre corrigés par la différence entre les temps de transfert des hydrogrammes depuis ces stations jusqu'à Montsoreau.

Toutes les valeurs de cette variable sont positives ou nulles, ce qui traduit bien le fait que les crues de la Vienne sont soit concomitantes à celles de la Loire, soit en avance.

L'histogramme de ces valeurs est porté sur la figure 1.

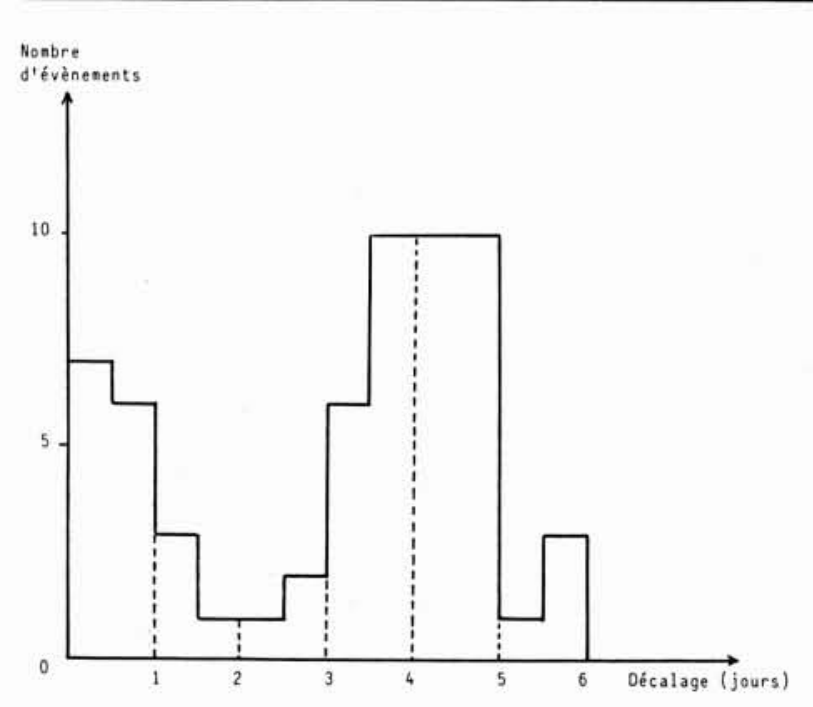

Figure 1 - Répartition des décalages entre les pointes de crues Loire amont - Vienne.
On peut distinguer deux cas bien différenciés :

- décalage de 0 à 1 jour, ce qui traduit une concomitance plus ou moins relative;

- crue de la Vienne en avance de 3 à 4,5 jours sur la crue de la Loire.

Les décalages inférieurs ou égaux à la journée concernent une douzaine de crues, soit environ $20 \%$ des cas.

Les cas (cas 1, exemple : figure 2) où la pointe de crue de la Vienne est en concomitance "parfaite" (décalage inférieur à la demi-journée) avec celle de la Loire, sont au nombre de 7 (soit $11 \%$ des cas): mars 1888 ( 2 crues), mars 1895, avril 1913, mars 1919, février 1928, mars 1931. Ils engendrent des événements à Montsoreau dont le débit de pointe ne dépasse pas $4500 \mathrm{~m}^{3} / \mathrm{s}$ et se réfèrent presque exclusivement au mois de mars. Ce sont principalement des événements liés au passage de plusieurs perturbations d'origine océanique à quelques jours d'intervalle. (Le bassin de la Vienne réagit plus rapidement aux précipitations tombant sur un sol déjà saturé).

La situation la plus fréquente ( $60 \%$ des cas environ) correspond à une avance de 3 à 4,5 jours du débit de pointe de la Vienne par rapport à celui de la Loire au niveau de la confluence.

Les risques de simultanéité des crues de la Loire et de la Vienne Analyse des événements de la période 1882-1979

Les causes météorologiques des événements rares observés sur la Loire conduisent à un classement " $a$ priori " en crues "méditerranéennes", "océaniques" et mixtes, regroupement confirmé par l'analyse typologique effectuée sur les 66 crues principales de la période 1882-1979 (analyse en composantes principales et nuées dynamiques).

Les crues importantes les plus fréquentes de la Loire à Montsoreau $(1910,1919,1923 \ldots)$ sont des crues de type " océanique classique " consécutives à des perturbations d'origine Ouest à Sud-Ouest produisant d'abondantes précipitations, en particulier sur les parties Nord-Ouest du Massif Central et le Haut Morvan. Ces crues sont formées par les apports de la Loire Moyenne et des affluents inférieurs (Cher, Indre, Vienne) : dans le cas où la crue est provoquée par le passage d'une unique perturbation, le maximum de la Vienne précède celui de la Loire du fait même du déplacement des perturbations océaniques et des caractéristiques des bassins versants concernés. Dans le cas du passage de plusieurs perturbations à quelques jours d'intervalle, on peut théoriquement aboutir à des concomitances possibles à la confluence.

L'origine des grandes crues catastrophiques du siècle dernier $(1846,1856,1866)$ est autre. Ce sont des crues de type " mixte ", c'est-à-dire formées par l'action simultanée de perturbations d'origine méditerranéenne de secteur Sud à Sud-Est intervenant sur la partie amont du bassin et de perturbations atlantiques qui étendent les précipitations à tout le bassin. 
Débit $(\mathrm{m} 3 / \mathrm{s})$

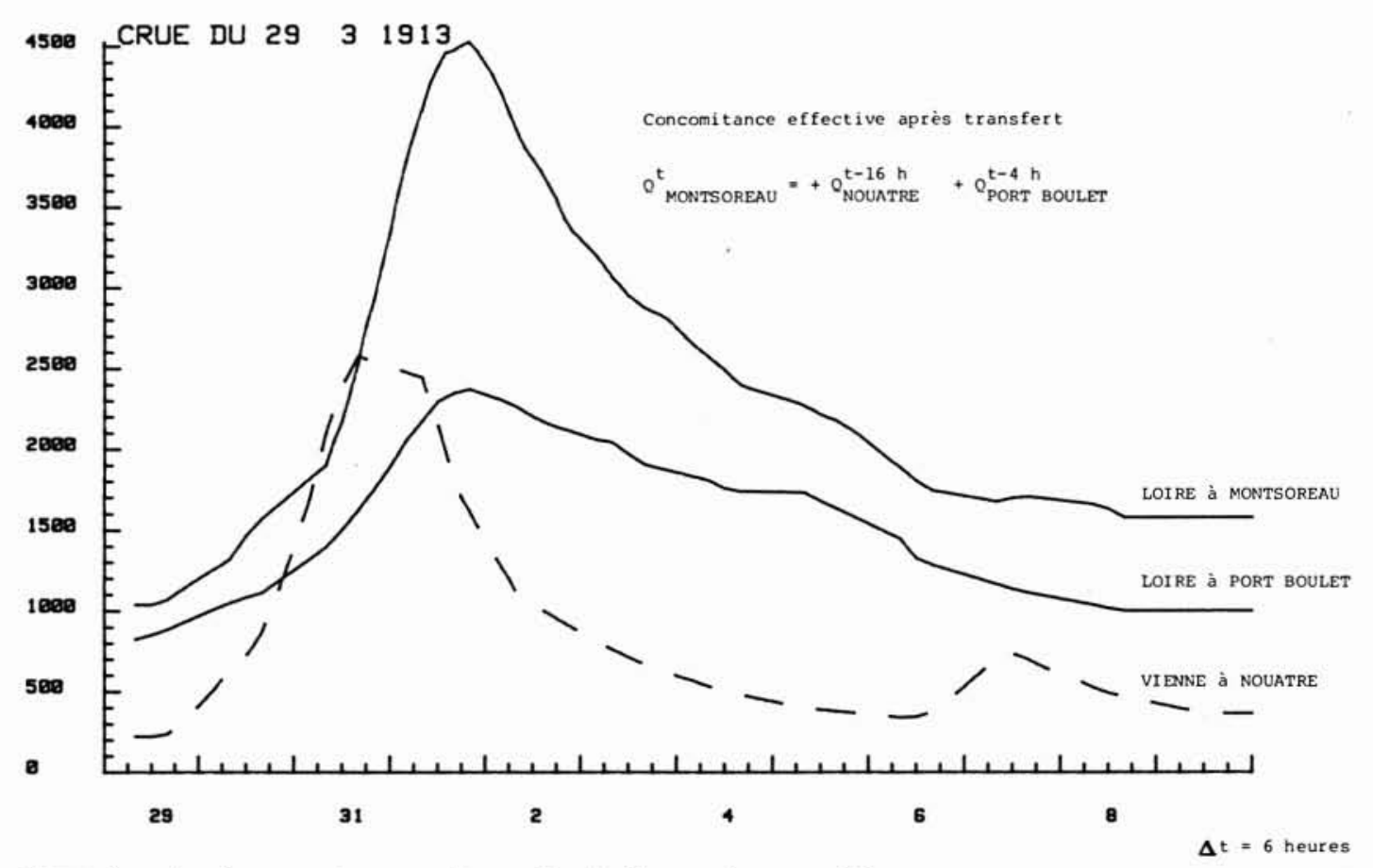

Figure 2 - Les deux cas de concomitance. Cas 1 : Concomitance « réelle».

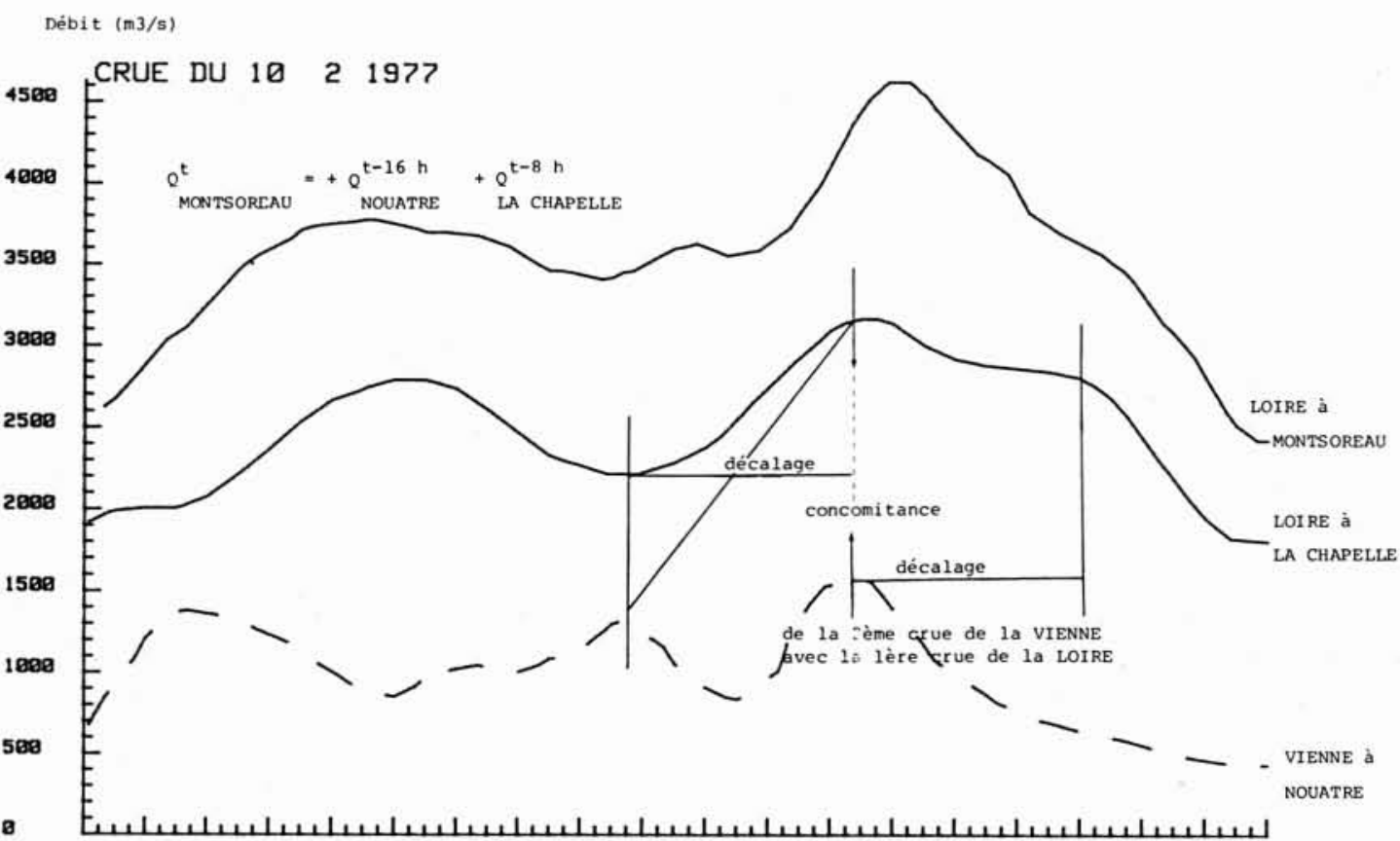
18
12
14
16
18
$28 \quad 22$
24
26
$\mathbf{2 8}$
$\mathbf{\Delta}=6$ heures

Figure 3 - Les deux cas de concomitance. Cas 2 : Concomitance par «rattrapage». 
Deux cas de figure peuvent être envisagés pour ce type de crues :

- la crue de la Vienne n'offre pas un caractère de gravité, l'influence méditerranéenne étant prépondérante. Les crues peuvent néanmoins figurer parmi les plus dévastatrices à hauteur de Montsoreau $(1866,1872)$, mais aucun aménagement sur le bassin de la Vienne ne permettra de modifier le caractère exceptionnel de ces événements;

- la crue de la Vienne est importante. Son maximum se produira généralement 3 à 4 jours avant le maximum de la crue de la Loire, mais si on envisage comme pour les crues de type océanique la possibilité d'une nouvelle onde de crue de la Vienne à 3 ou 4 jours d'intervalle de la première, une concordance des maximums à la confluence de la Loire et de la Vienne pourrait survenir et conduirait alors à un événement extraordinaire dans le cas d'événements rares sur la Vienne et sur la Loire.

A l'intérieur de cette population, on peut imaginer une situation critique correspondant à une succession de crues d'origine atlantique sur la Vienne avec une périodicité de 3 à 4,5 jours. Une crue de la Vienne pourrait alors se trouver en concomitance avec une crue antérieure de la Loire amont (Cas 2, exemple : figure 3). Cette conjonction, dans le cas de deux crues d'importance sur chaque branche, pourrait conduire à des événements rares. Quatre événements (soit $6 \%$ des cas) présentent cette caractéristique de concomitance par "rattrapage " d'une crue antérieure de la Loire par une crue de la Vienne : février 1897, mars 1906, février 1955 et février 1977. Ils engendrent des débits à Montsoreau qui sont relativement élevés (période de retour de 5 à 10 ans), mais en aucun cas, nous ne sommes en présence d'une conjonction de l'influence des vents pluvieux de secteur Sud-Sud Est sur le haut bassin de la Loire et de celle des vents atlantiques du secteur Ouest à Sud-Ouest.

Finalement, la concomitance vraie ou par rattrapage apparaît dans $7+4=11$ cas sur les 66 événements les plus forts observés sur 98 années. Aucun de ces cas ne se refère à une combinaison d'averses méditerranéennes et océaniques qui, décalées dans le temps, auraient pu provoquer une concomitance au niveau de la confluence LoireVienne. L'examen des distributions des débits maximaux des crues avec et sans concomitance, montre par ailleurs que cette concomitance n'est pas un facteur explicatif des plus fortes valeurs observées à montsoreau sur l'échantillon étudié. (Le taux moyen de participation de la Vienne dans les crues à Montsoreau est de $30 \%$ aussi bien en débit de pointe qu'en volume).

\section{Efficacité sur les crues de la Loire à Montsoreau d'un réservoir d'écrêtement sur la Vienne, en aval du confluent de la Creuse}

Examiner la concomitance des crues de la Vienne et de la Loire conduit à s'interroger sur la façon d'intervenir pour éviter ou diminuer ce phénomène et atteindre un certain degré de protection.

Les moyens disponibles correspondants sont essentiellement la construction d'ouvrage écrêteur sur la Vienne et (ou) sur la Loire et l'Allier. L'étude de l'efficacité des barrages du Veurdre et de Villerest étant réalisée par ailleurs, il restait à examiner les possibilités d'un barrage écrêteur sur la Vienne aval.

L'étude sommaire qui a été réalisée pour tester l'efficacité sur les crues de la Loire à Montsoreau d'un réservoir d'écrêtement situé sur la Vienne à Nouatre, en aval du confluent avec la Creuse, consiste en une simulation du fonctionnement hydrologique du système avec un réservoir écrêteur géré de façon optimale (règles de gestion et prévision parfaites): les hydrogrammes sont écrêtés dans leurs valeurs supérieures jusqu'à concurrence du volume retenu du réservoir (scénarios à 50,100 et $150 \mathrm{Mm}^{3}$ ).

Bien que simple, cette approche permet de définir l'ordre de grandeur des améliorations obtenues sur les crues de la Loire en fonction du volume d'une retenue sur la Vienne.

Si l'on fait abstraction du problème, réel et non résolu, d'emplacement du site de barrage correspondant, les résultats obtenus se situent en amont des choix économiques entre coûts de rehaussement des digues sur la Loire et coûts de construction d'un ouvrage sur la Vienne.

Les résultats obtenus sur la période 1882-1979 conduisent finalement à s'interroger sur l'opportunité de réaliser un tel ouvrage et à situer son coût par rapport à celui du rehaussement des levées et des digues.

Les conséquences d'une retenue de $150 \mathrm{Mm}^{3}$ de volume efficace se limitent en moyenne à un gain de l'ordre d'une trentaine de $\mathrm{cm}$ (environ $400 \mathrm{~m}^{3} / \mathrm{s}$ ). La saison intervient peu et les effets sur les événements les plus extrêmes atteignent $75 \mathrm{~cm}$ (crue centennale) (Fig. 4 à 7).

\begin{tabular}{|c|c|c|c|c|}
\hline \multirow[b]{2}{*}{$\begin{array}{lr}\text { Débits } & \\
\text { maximaux } & \text { Moyenne } \\
\text { de la Loire } & \mathrm{m}^{3} / \mathrm{s} \\
\text { à Montsoreau } & \text { Ecart-type }\end{array}$} & \multicolumn{4}{|c|}{$\begin{array}{l}\text { Volume d'écrêtement } \\
\text { sur la Vienne }\end{array}$} \\
\hline & $\begin{array}{r}3599 \\
906\end{array}$ & $\begin{array}{r}3403 \\
846\end{array}$ & $\begin{array}{r}3275 \\
831\end{array}$ & $\begin{array}{r}3164 \\
824\end{array}$ \\
\hline Gain en débit $\left(\mathrm{m}^{3} / \mathrm{s}\right)$ & & 196 & 324 & 435 \\
\hline Gain en hauteur $(\mathrm{cm})$ & & 13 & 26 & 36 \\
\hline
\end{tabular}

Encore faut-il souligner que ces gains sont obtenus à partir d'un schéma de gestion optimale sans doute assez éloigné des possibilités de gestion en temps réel. Compte tenu des problèmes de prévision hydrologique, de délai de vidange de l'ouvrage, d'éventuelles crues estivales ou de peu probables crues successives, d'impossibilité "sociale " de vider totalement la retenue en période d'été,..., il faudra envisager un volume utile de la retenue beaucoup plus important que $150 \mathrm{Mm}^{3}$ pour limiter au maximum des risques de défaillance (rappelons à titre d'exemple que le rapport volume utile/volume total ne dépasse pas $50 \%$ pour le barrage de Veurdre); or, la recherche d'un site de construction en aval de Nouatre est limité par la zone d'influence du remou de la Loire, qui représente une importante possibilité de stockage.

L'importance de l'investissement ne semble pas pouvoir être compensée par une mise en valeur optimale de l'ouvrage : le délai de prévision sur la Vienne n'excède pas 24 heures, il paraît difficilement envisageable de rentabiliser cette vidange préalable en termes énergétiques ou de soutien d'étiage. 


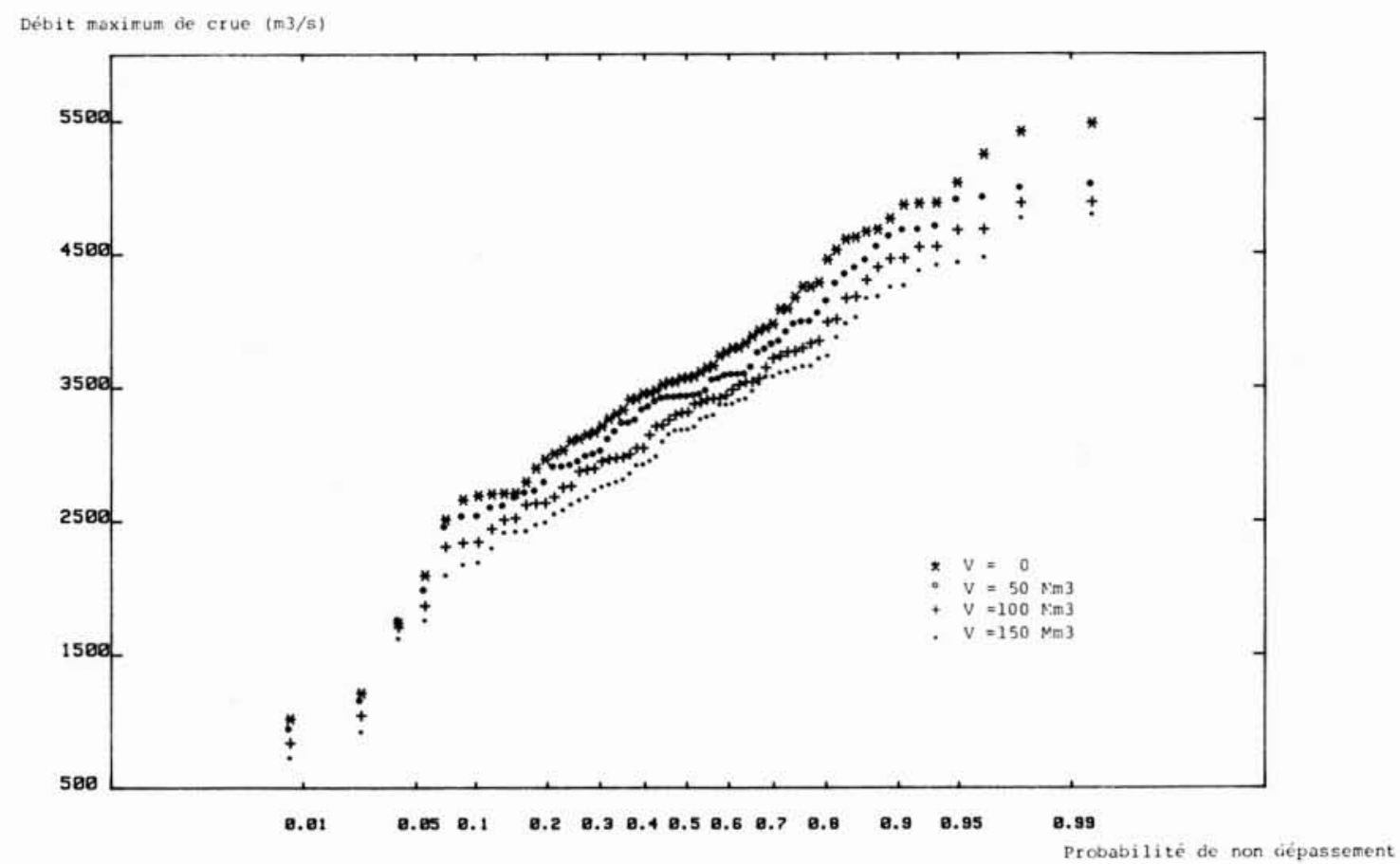

Figure 4 - Distribution des débits maximaux de la Loire à Montsoreau selon le volume d'un barrage écrêteur sur la Vienne.

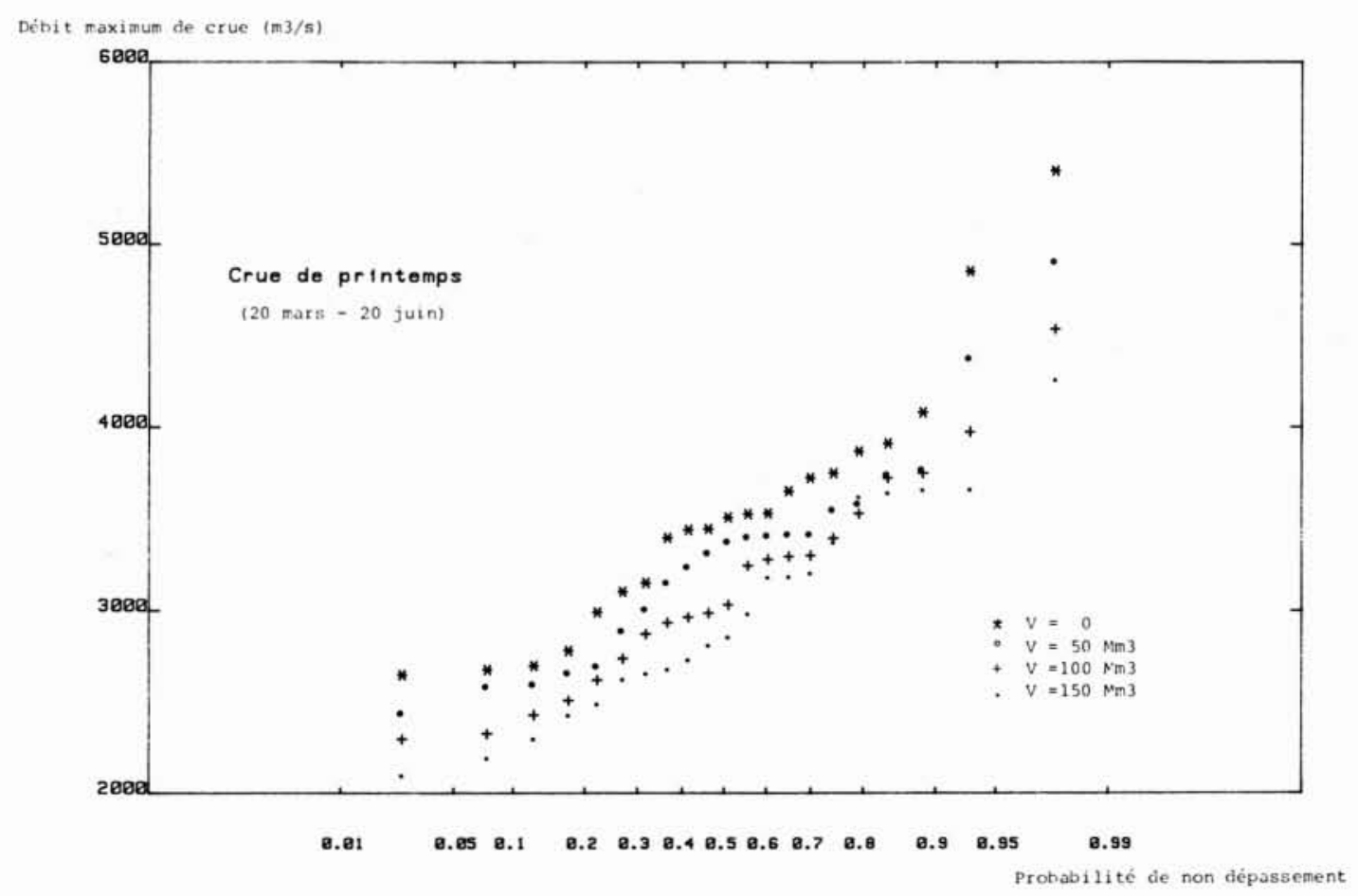

Figure 5 - Distribution par saison des débits maximaux de la Loire à Montsoreau selon le volume d'un barrage écrêteur sur la Vienne. 


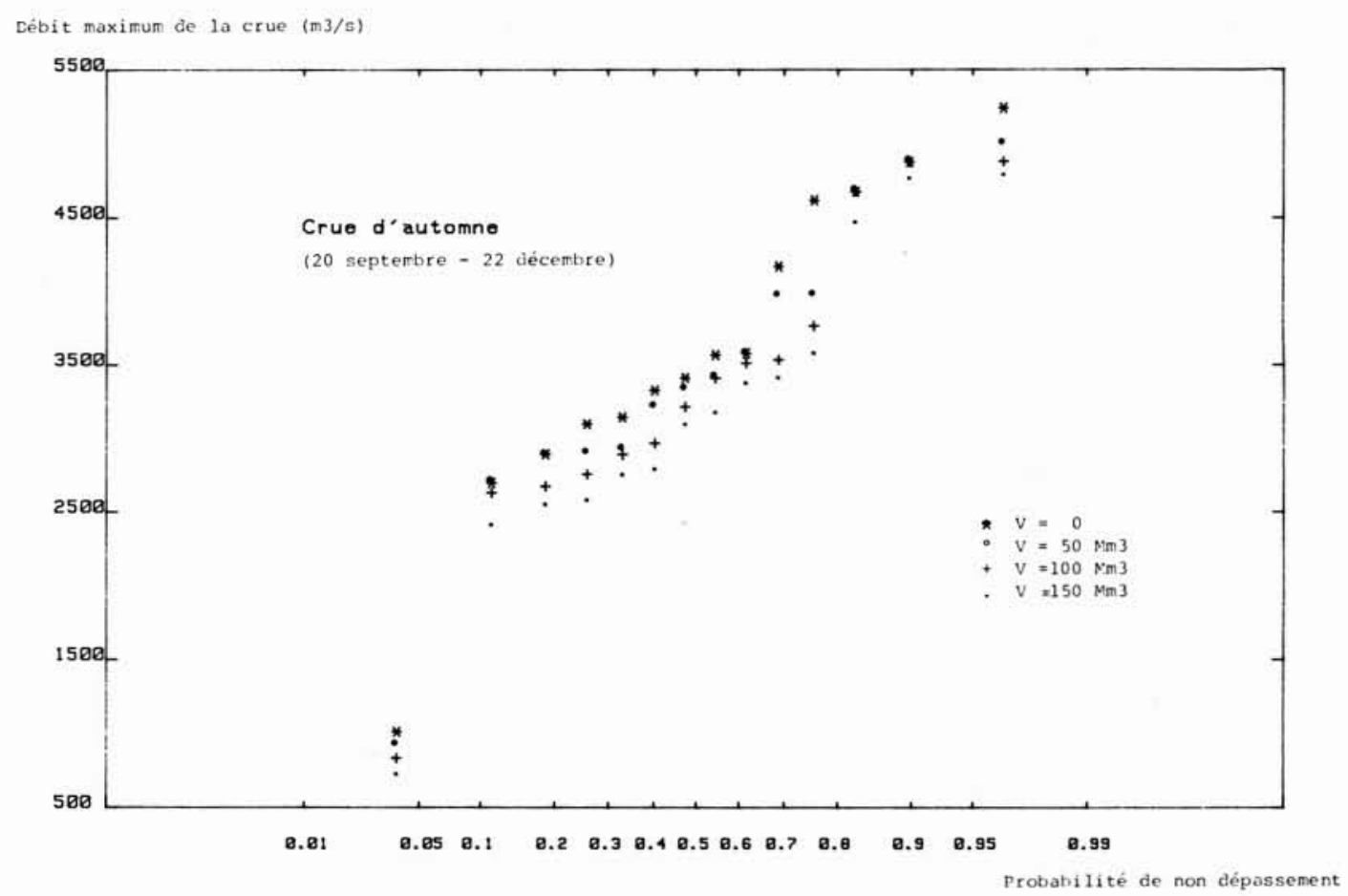

Figure 6 - Distribution par saison des débits maximaux de la Loire à Montsoreau selon le volume d'un barrage écrêteur sur la Vienne.

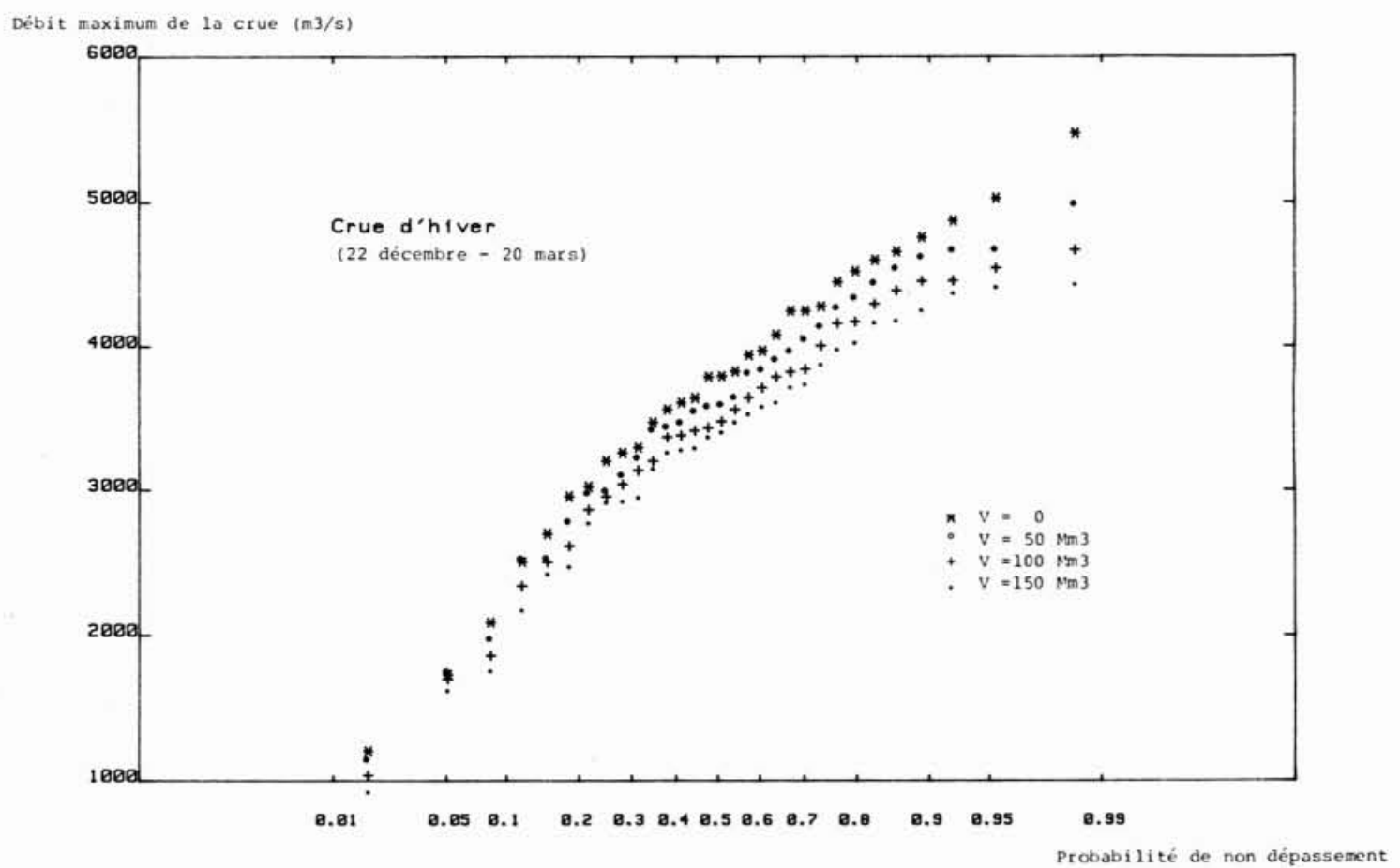

Figure 7 - Distribution par saison des débits maximaux de la Loire à Montsoreau selon le volume d'un barrage écrêteur sur la Vienne. 


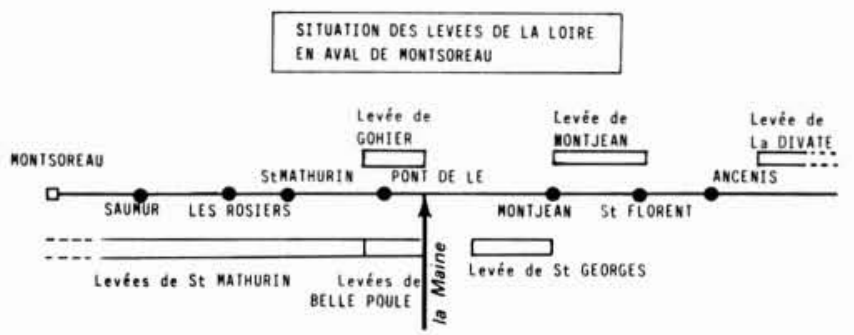

Figure 8
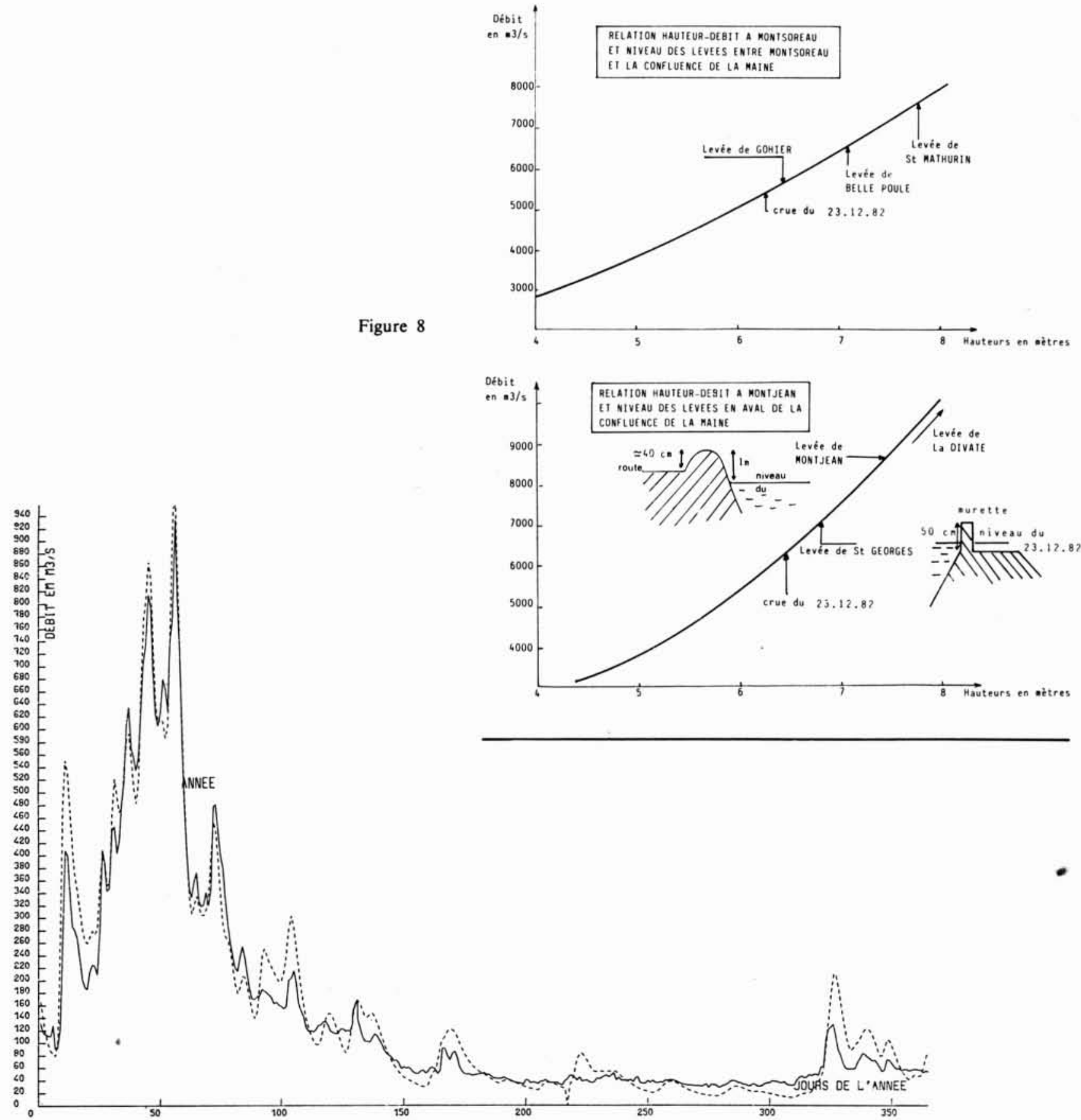

Figure 9 -Débits journaliers: Débits de la Maine à Angers calculés avec les débits de ses affluents.Traits pleins = Débits observés Traits pointillés $=$ Débits calculés 
M3/s

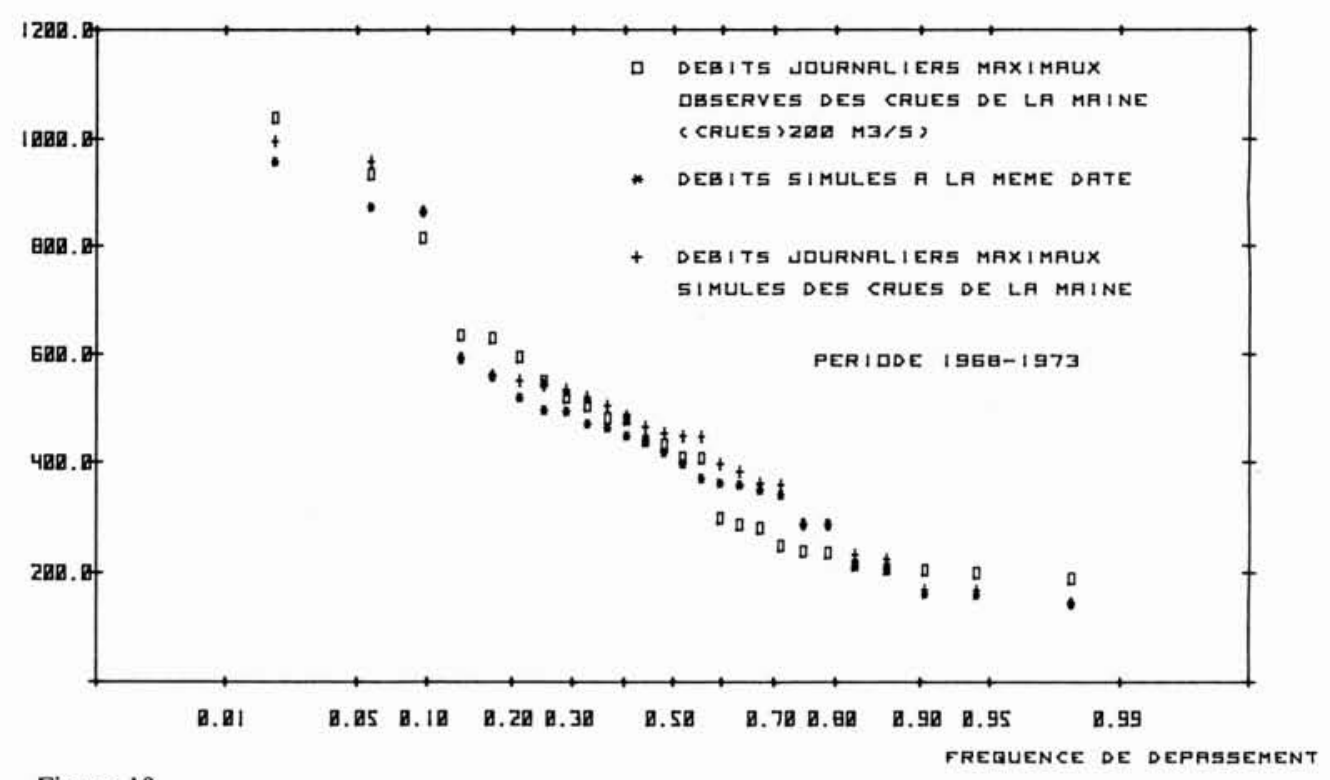

Figure 10

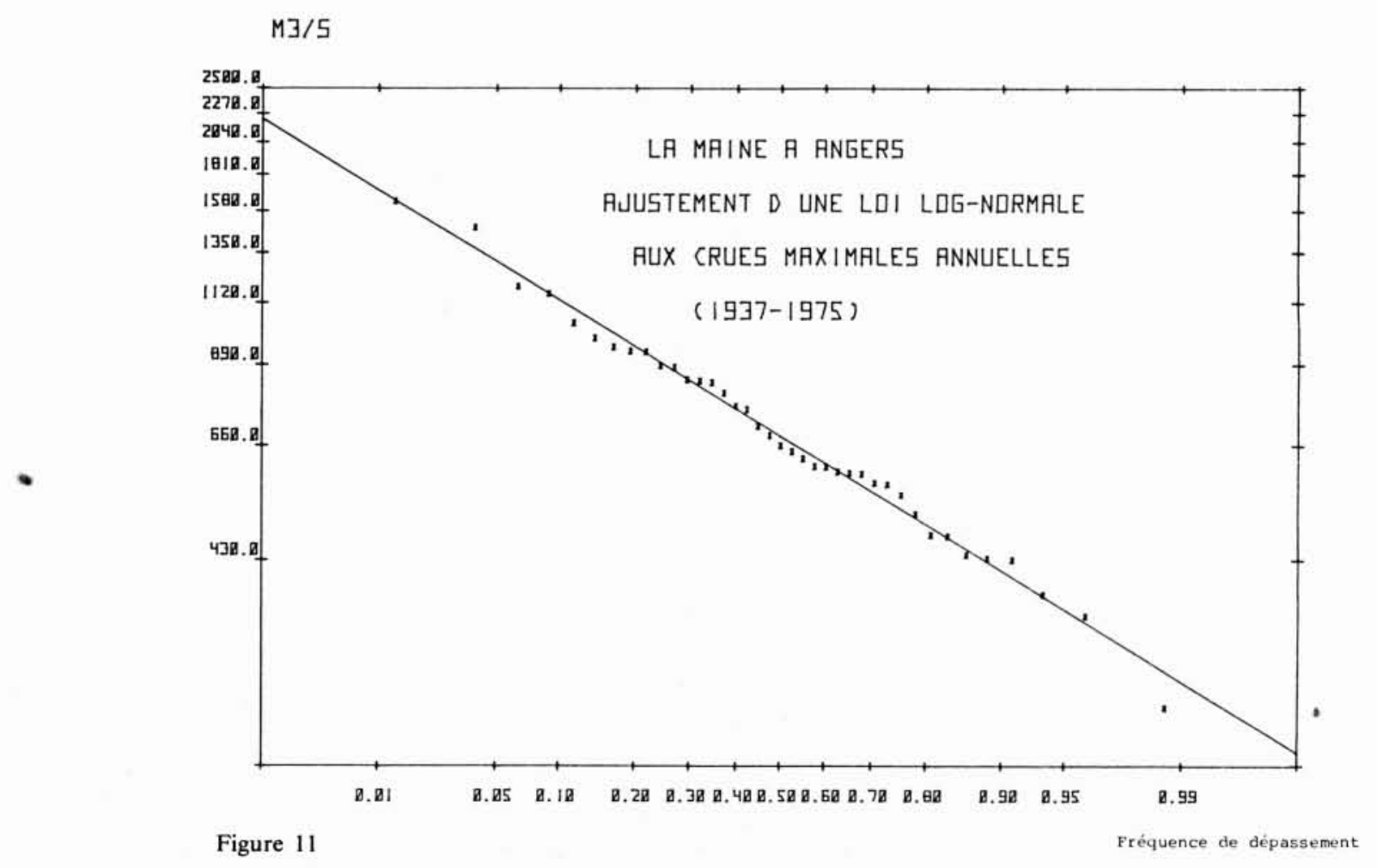


On se trouve donc face à deux choix :

- projeter un ouvrage de $300 \mathrm{Mm}^{3}$ pour gagner en moyenne $30 \mathrm{~cm}$ sur le niveau de la Loire en aval de la confluence tout en n'assurant pas une protection totale puisque les crues de la Loire amont ne sont pas concernées;

- ou se limiter à un rehaussement en aval de la confluence, intervention beaucoup moins coûteuse, et efficace pour toutes les crues quelle que soit leur origine.

Les risques de submersion des levées sur la Loire en aval du confluent de la Vienne

La crue de décembre 1982, survenue en fin d'étude, n'apparaît pas dans l'échantillon traité. De période de retour élevée (du même ordre que la crue de 1910), elle a atteint $5400 \mathrm{~m}^{3} / \mathrm{s}$ à Montsoreau et fait partie des crues présentant une certaine simultanéité entre crues de Vienne et de Loire. Ses caractéristiques n'auraient certainement pas modifié les conclusions de ce rapport, mais le niveau d'eau atteint permet de juger du degré de protection fourni par les levées (Fig. 8).

En aval de la Vienne, les levées ont présenté jusqu'à La Daguenière, un niveau de protection très confortable, de l'ordre de $1,50 \mathrm{~m}$ (protection du val d'Authion sur une soixantaine de $\mathrm{km}$ ).

La situation est apparue un peu dégradée pour la levée de la Belle Poule; le rehaussement du lit de la Loire de plus d'un mètre qui s'est produit depuis le siècle dernier, a conduit à une marge de sécurité de l'ordre de 60 à 80 $\mathrm{cm}$ seulement. En rive gauche, la protection est apparue encore plus faible, puisque la hauteur maximale de la crue s'est trouvée à $15 \mathrm{~cm}$ du niveau des levées !

En aval de la Maine, l'efficacité de la levée de Saint-Georges en rive droite n'a été due qu'à la présence d'une murette de quelques $50 \mathrm{~cm}$ de hauteur par rapport à la route !

La situation est nettement meilleure au niveau de la levée de Montjean et de la Divate.

Si ces éléments permettent de définir une politique d'aménagement et de rehaussement complémentaires des levées au niveau des points sensibles, on n'a par contre aucune certitude sur l'état de ces levées, en particulier à hauteur des villes.

Il paraît donc nécessaire de prévoir, parallèlement aux travaux de rehaussement, une campagne de contrôle et d'entretien et ce, sur l'ensemble des levées.

\section{Risque de submersion \\ des Vals de Loire situés en aval de la confluence de la Maine par simulation des débits des sous-bassins}

L'étude du risque de submersion des vals situés en aval du confluent de la Maine est limitée par l'absence de contrôle ancien sur cette rivière.
Les débits de la Maine, en fait estimés par sommation des observations recueillies sur les divers affluents (Oudon, Mayenne, Sarthe et Loir) ne sont disponibles que depuis 1968.

Afin d'obtenir une information suffisante, nous avons valorisé les longues séries de pluviométrie journalières régionales existantes, par établissement d'une relation pluie-débit sur la période d'observation concomitante. Malgré l'importance des superficies concernées, rendant généralement ce type d'approche inadaptée, un modèle global conceptuel (CREC) a été appliqué aux quatre bassins versants des principaux affluents, les débits de la Maine étant ensuite estimés par addition des séries simulées (Fig. 9 et 10).

L'analyse de la série simulée + observée (1937-1978) conduit à des estimations en ce site d'un débit décennal de l'ordre de $1100 \mathrm{~m}^{3} / \mathrm{s}$ et d'un débit centenaire proche de $1700 \mathrm{~m}^{3} / \mathrm{s}$ (Fig. 11).

Les événements correspondants ont été confrontés à ceux de la série observée sur la Loire à Montsoreau, ce qui a permis de chiffrer la part d'intervention des crues de la Maine sur le débit de la Loire, à l'aval de la confluence et de réaliser une rapide analyse fréquentielle de la concomitance.

L'examen de la simultanéité des crues de la Loire et de la Maine abordé de manière simple au niveau des débits maximaux, montre que dans le cas d'une crue sur la Loire à Montsoreau, la part d'intervention de la Maine dans le

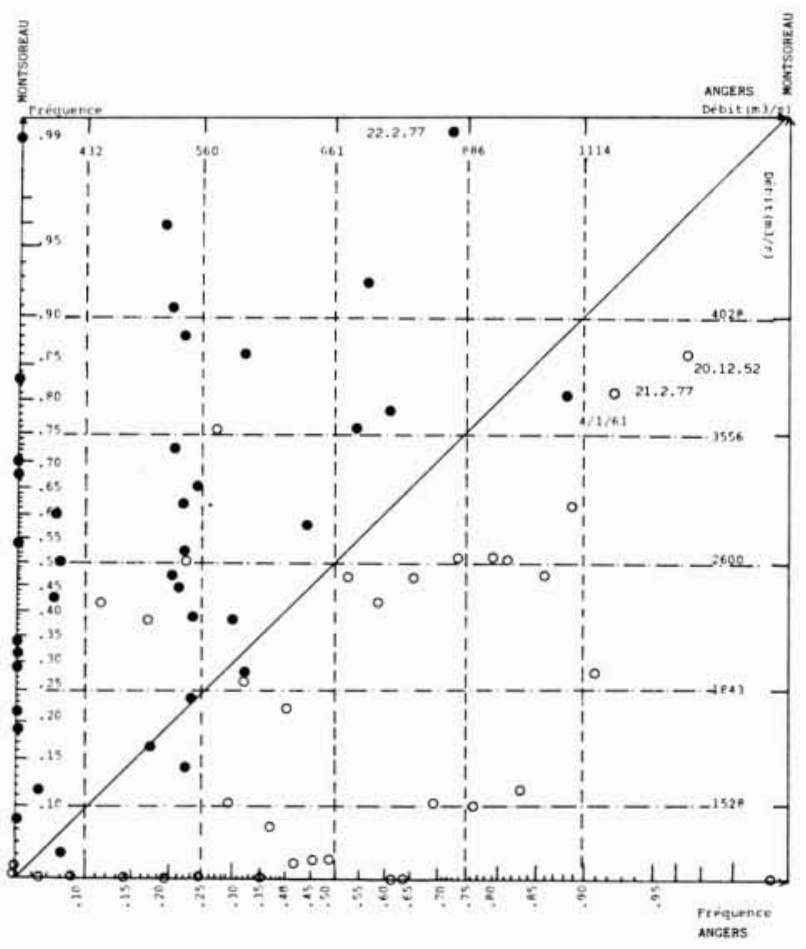

Figure 12 - Concomitance des crues de la Maine à Angers et de la Loire à l'aval de la confluence.

Représentation fréquentielle - Période 1937-1975.

- Crue maximale annuelle et correspondance à Angers au jour $j+1$

Crue maximale annuelle et correspondance à Montsoreau au jour $j-1$. 


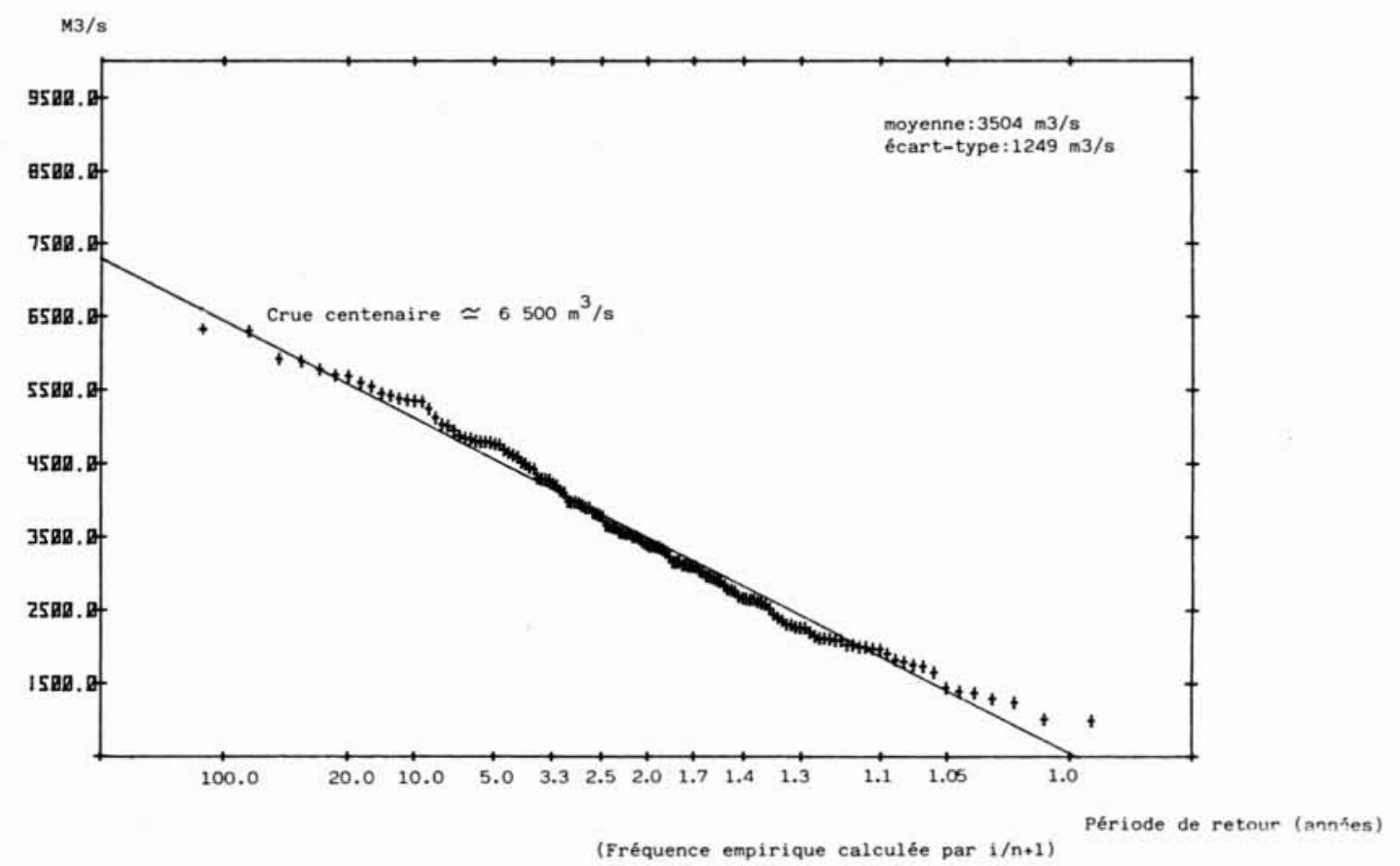

Figure 13 - La Loire à Montjean.

Ajustement d'une loi normale aux débits maximaux instantanés annuels (1843-1983).

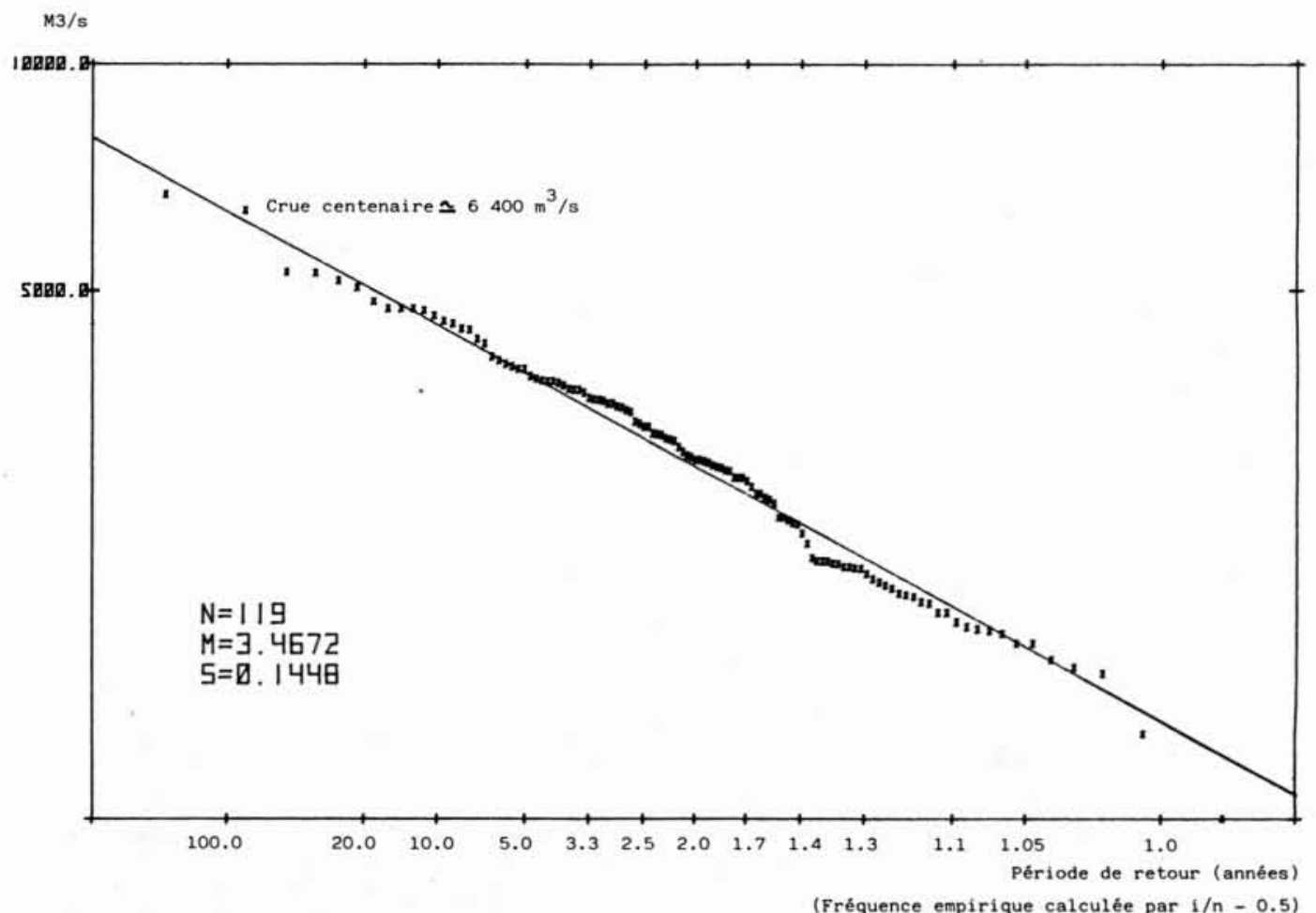

Figure 14 - La Loire à Monstoreau.

Ajustement d'une loi log-normale aux débits maximaux annuels (1856-1983) 
débit maximal à l'aval de la confluence ne dépasse pas $15 \%$ en moyenne et reste de toute façon inférieure à $30 \%$.

Ces résulats sont pratiquement identique dans le cas d'une crue de la Loire à Montjean.

Dans le cas d'une crue de la Maine (il y a bien sûr recouvrement d'une partie de ces différents scénarios), la part d'intervention sur le débit de la Loire est supérieur à $30 \%$ en moyenne, atteignant $60 \%$ au maximum (crue de novembre 1966).

La représentation sur un même graphique des crues maximales annuelles de chacune des rivières, accompagnées du débit correspondant sur l'autre rivière $\left(\mathrm{en} \mathrm{m}^{3} / \mathrm{s}\right.$ ou en fréquence) permet de juger du degré de concomitance des événements extrêmes; si les points s'organisent selon la première bissectrice, droite d'équi-fréquence, on aura simultanéité. Appliqué au cas de la Maine et de la Loire, ce type de représentation montre une grande dispersion: seules quelques rares crues présentent des fréquences de même ordre, mais en aucun cas le débit de la Maine n'excède $30 \%$ du débit de la Loire à l'aval de la confluence (Fig. 12).

Finalement, les risques de submersion en aval de la confluence dus à la simultanéité des crues de la Loire et de la Maine sont extrêmement faibles, la part de la Maine ne représentant qu'une fraction minime des débits à l'aval de la confluence.

Aucun aménagement sur la Maine (le site resterait à trouver...) n'aura de conséquence notable sur les risques de submersion en aval de la confluence avec la Loire.

\section{Conclusion}

L'idée initiale de la protection des Vals de la Loire en aval de la confluence de la Vienne par réalisation d'un barrage écrêteur de crue, paraît difficile à maintenir.

Un tel ouvrage, d'un volume au moins égal à $300 \mathrm{Mm}^{3}$ (c'est-à-dire d'un coût supérieur à 1 milliard de francs !...) ne fournirait, dans le cas d'un schéma de gestion optimale, qu'un gain de $30 \mathrm{~cm}$ en moyenne sur les hauteurs de la Loire à Montsoreau.

Moins de $30 \%$ des crues à Montsoreau sont consécutives à une certaine concomitance des crues de la Loire et de la Vienne.

C'est dire, en d'autres termes, qu'un tel ouvrage n'assurerait pratiquement aucune protection dans plus de $70 \%$ des cas!

Si l'on rajoute à ces résulats les difficultés d'implan- tation d'un tel ouvrage, compte tenu de la nécessité de préserver l'importante zone de stockage naturel des Vals de la Vienne, la protection des Vals de Loire en aval du confluent de la Vienne semble devoir passer par d'autres choix.

La crue de décembre 1982 a montré l'efficacité des levées existantes, mais aussi leur faiblesse en certains points. Une politique de renforcement et de rehaussement des levées augmenterait l'efficacité de ces ouvrages qui offrent le double avantage :

- d'assurer une protection pour l'ensemble des crues quelle que soit leur origine;

- de présenter des coûts de construction nettement plus réduits qu'un barrage écrêteur.

\section{Bibliographie}

\author{
MIQUEL (1964) \\ Calage des plate-formes des centrales nucléaires. Estimation de \\ la crue millénaire de la Loire à Port Boulet et à Montjean. \\ Rapport EDF C 45/74.12. \\ PARDÉ (1964) \\ Etudes potamologiques sur la Loire et ses affluents. \\ NOROIS $\mathrm{n}^{\circ} 44$ bis $\mathrm{II}^{e}$ année. \\ JACQUET (1964) \\ Note sur les possibilités d'atténuation des crues de la Loire à \\ l'aval du Bec du Cher. \\ Rapport EDF-CREC HYD 10.64. \\ SOGREAH (1979) \\ Etude hydrologique des crues de la Vienne. \\ Rapport 361230 - Décembre 1979.
}

J.M. BELLOSTAS, A. GUILBOT (1982)

Etude du risque de submersion en aval de la confluence de la Maine par simulation des débits.

Rapport 34/82 du Laboratoire d'Hydrologie Mathématique, U.S.T.L. Montpellier.

A. GUILBOT, J.C. HEMAIN, P. RAOUS, Y. TRÉVISIOL (1982) Etude et estimation du risque de simultanéité des crues de la Loire et de la Vienne au niveau de leur confluence. Rapport 38/82 du L.H.M., U.S.T.L. Montpellier.

J.M. BELlostAs, A. GUILBOT, J.C. HÉMAIN, P. RAOUS (1982)

Efficacité d'un barrage écrêteur par la Vienne.

Rapport 31/82 du L.H.M., U.S.T.L. Montpellier. 


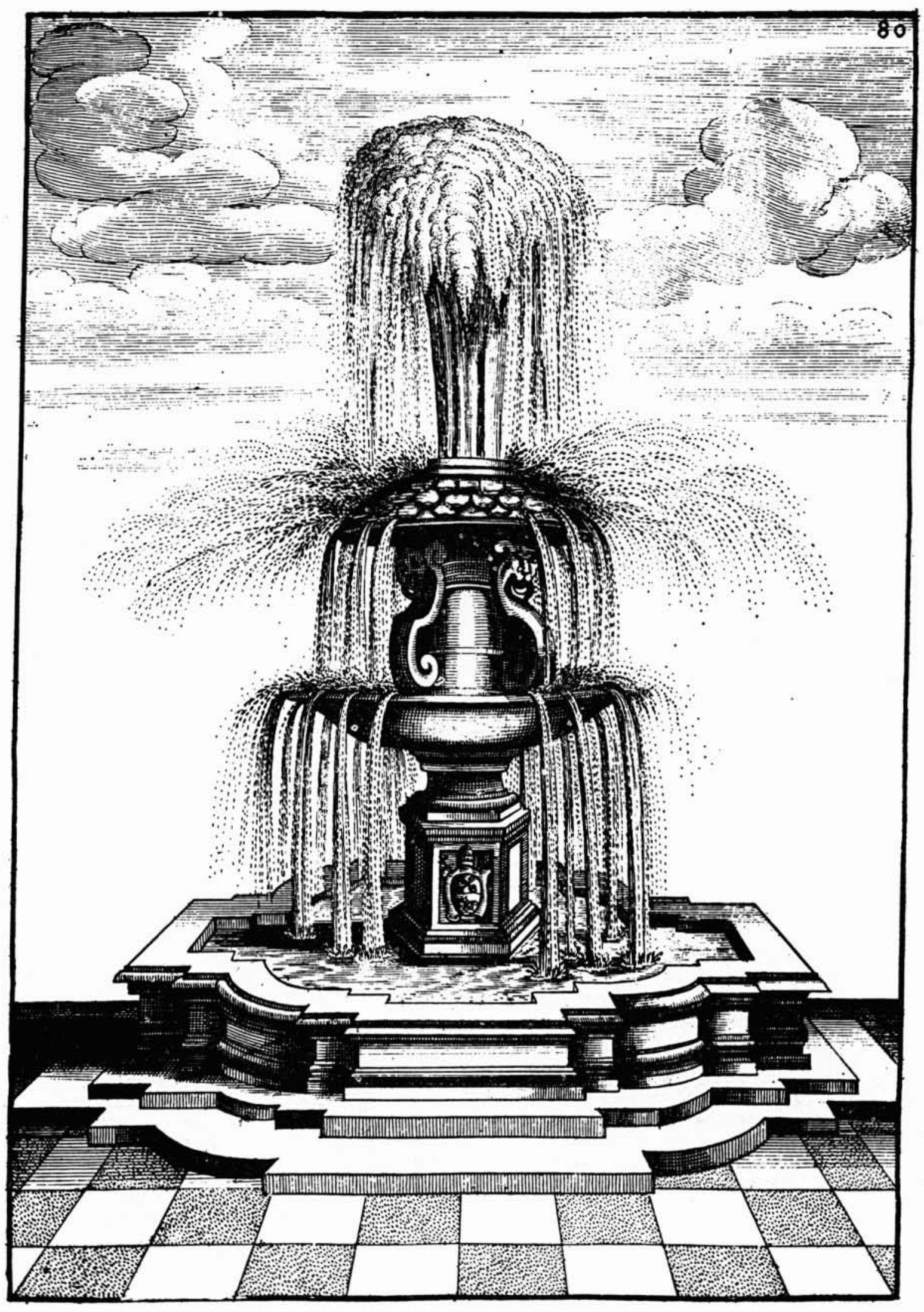

